\title{
Planeación transfronteriza: ¿un caso de provocación sofisticada?
}

\author{
John Friedmann \\ Rebeca Morales \\ Universidad de California-Los Ángeles
}

\section{Resumen}

Este documento se propone examinar críticamente los argumentos de Hansen a favor de un marco formal y legalmente comprometido, para la planeación transfronteriza y cooperación en la frontera de Estados Unidos y México. Primero se procederá a resumir los argumentos de Hansen favorables para una cooperación transfronteriza más estrecha. Tres conjuntos de argumentos serán discutidos: aquellos relacionados con su concepción de regiones fronterizas como "artificialmente" divididas por convenciones políticas; aquellos relacionados con la autonomía regional; y aquellos relacionados con la cooperación económica internacional. Siguiendo con esta revisión, se trabajará sobre lo que se consideran las falacias básicas en el razonamiento de Hansen, concluyendo que su propuesta para una estrecha cooperación transfronteriza, basada en sus ejemplos europeos, debe ser rechazada.

Palabras clave: frontera, interdependencia económica, planeación transfronteriza, zona de libre comercio, dimensiones contrastantes.

\begin{abstract}
This document pretends to critically consider Hansen's arguments that help to create a formal and legally committed structure, for an efficient trans-border planning and cooperation at the border of Mexico and the United States. On first place, it will make a summary of Hansen's arguments that fosters the closer trans-border cooperation. Three groups of arguments will be discussed: those related to the conception of border regions as "artificially" divided by political regulations; those related to the regional autonomy; and those regarding to the international economic collaboration. This revision will continue working on what is considered basic fallacies of Hansen's ideas. Finally, it will get to the conclusion that his proposal for a jointly trans-border cooperation, based on his European examples must be rejected.
\end{abstract}

Keywords: border, economic interdependence, trans-border planning, free trade zone, contrasting dimensions. 


\title{
PLANEACION TRANSFRONTERIZA: ¿UN CASO DE "PROVOCACION SOFISTICADA"?* 1
}

\author{
Por \\ John Friedmann \\ Rebeca Morales \\ University of Ca lifornia, Los Angeles
}

En una publicacion reciente del Journal of the American Institute of Planners, el eminente economista regional, Niles Hansen, discute el caso de lo que él llama "cooperación transfronteriza" en la frontera México-Estados Unidos $^{2}$ Sostiene que los esfuerzos actuales en el tratamiento de un "conjunto de problemas sociales, económicos y ambientales que requieren cooperación transfronteriza" son "sumamente informales y por tanto fragmentarios y asistemáticos", concluyendo con un llamado para un enfoque internacional: ${ }^{3}$

"A la luz de la experiencia europea parece razonable para los Estados Unidos y México cuando menos explorar las posibilidades de creación (aún sobre una base experimental) de acuerdos, estatutos o contratos de cooperación transfronteriza entre sus respectivas comunidades o regiones fronterizas. Discusiones similares podrían ser emprendidas por Estados Unidos y Canadá, aunque los problemas. .."

Hansen hace referencia a seis cstudios de caso de Europa occidental, los cuales constituyen la base de su artículo. Ellos son brevemente resumidos a continuación:

1. Regio Basiliensis es una región fronteriza centrada en Basilea que abarca Suiza, Francia y Alemania. Establecida en 1963, esta asociación regional se ha dedicado principalmente a asuntos de transporte y ambientales. Entre sus más importantes logros está la construcción de un aeropuerto regional en Francis.

2. Euregio, otro compacto regional, fue establecido en 1970. En él se incluyen tres asociaciones autónomas de municipalidades a lo largo de la frontera germano-holandesa. Euregio tiene un consejo clecto que desarrolla programas y políticas comunes en cuestiones cconómicas y socioculturales.

*Trabajo presentado ante la Conferencia de Estudios Fronterizos, Tijuana, B. C., abril 27 de 1984. Traducción al español por el M.C. Agustín Sández Pérez.

'La frase "provocación sofisticada" ha sido atribuida a Jesús Puente Leyva, miembro de la Cámara de Diputados de México.

${ }^{2}$ Hansen, Niles, 1983.

3 Ibidem. 
3. La Conferencia de Planificadores del Valle del Alto Rhin, es un esfuerzo reciente por promover planeación transfronteriza sobre una escala geográfica relativamente mayor. Su área de influencia se extiende de Basilea al norte de Frankfurt, sin embargo, la mayoría de las organizaciones participantes son actualmente de Alemania Occidental. La conferencia ha enfatizado cuestiones transfronterizas que afectan a Alemania Occidental, Francia y Suiza, con un énfasis en cuestiones ambientales y de uso de suelo.

4. Dentro del Consejo Europeo está la Conferencia Europea de Ministros responsables de la Planeación Regional y los relacionados con la Conferencia de Autoridades Locales y Regionales de Europa (CLRAE). Esta última organización, de acuerdo con Hansen, es el "único cuerpo en Europa que representa oficialmente a autoridades locales y regionales en sus relaciones con instituciones internacionales. . .". La conferencia "enfoca las relaciones transfronterizas como un proceso que potencialmente podría desplazarse sobre cinco fases crecientemente complejas: intercambio de información, consulta recíproca, cooperación activa, armonización de la planeación, e integración de la planeación" 5 .

5. La Asociación de Regiones Fronterizas Europeas, con sede en Bonn, es un "vehículo para el intercambio de experiencias y la promoción de la cooperación transfronteriza" ${ }^{6}$. Sus miembros incluyen 15 organizaciones regionales, la gran mayoría del Valle del Rhin. (Una organización similar, el Comité para la promoción de Cooperación Regional Alpina, se localiza en Turín, Italia). Fstas entidades, nos informa Hansen, son a su vez miembros de la Oficina Liaison de Organizaciones Regionales Europeas la cual se localiza en Estrasburgo (Francia) y constituyen un vínculo entre el Consejo de Europa (y particularmente el CLRAE) y los variados esfuerzos de cooperación transfronteriza del continente europeo. ${ }^{7}$

6. La Convención Limitrofe Europea sobre Cooperación Transfronteriza cntre Comunidades o Autoridades Territoriales, desarrollado entre 1975 y 1979 dentro del Consejo de Europa, fue abierto para su firma en Madrid en mayo 21 de 1980. Como convención ejecutiva, establece el marco legal para acuerdos transfronterizos locales y regionales. Es específicamente este marco el que Hansen tiene en mente al defender "acuerdos, estatutos o contratos sobre cooperación transfronteriza entre respectivas regiones o comunidades fronterizas" como se asentó en nucstra referencia inicial.

En este documento, nos proponemos examinar críticamente los argumentos de Hansen en favor de un marco formal y legalmente comprometido, $\overline{4}$ Ibidem.
${ }^{5}$ Idem.
${ }^{6}$ Idem.
${ }^{7}$ Idem. 
para la planeación transfronteriza y cooperación en la frontera de Fstados Unidos y México. Procederemos inicialmente resumiendo los argumentos de Hansen favorables para una cooperación transfronteriza más estrecha. Tres conjuntos de argumentos serán discutidos: aquellos relacionados con su concepción de regiones fronterizas como "artificialmente" divididas por convenciones políticas; aquellos relacionados con la autonomía regional; y aquellos relacionados con la cooperación económica internacional. Siguiendo con esta revisión, trabajaremos sobre lo que nosotros consideramos las falacias básicas en el razonamiento de Hansen, concluyendo que su propuesta para una estrecha cooperación transfronteriza, basada en sus ejemplos europeos, debe ser rechazada.

\section{EL CASO DE COOPERACION TRANSFRONTERIZA}

El concepto de un límite artificial. Es significativo, pensamos, que Hansen tienda a utilizar el término neutral de límite (boundary) -referido a línea que indica un límite- a cambio del rico, aunque más ambíguo concepto de frontera, que se refiere, como nos dice el diccionario, a un distrito o región que ocupa cada lado de una línea limítrofe. Las líncas limítrofes son lineales, y como fenómeno político, tratan en parte de impedir el flujo de bienes y de factores de la producción lo que, en la concepción neoclásica - concepción que mantiene lo político como fundamentalmente irracionales visto como necesario para alcanzar altas tasas de crecimiento económico. En el razonamiento neoclásico, las fronteras de la eficiencias reemplazan las fronteras políticas. El libre movimiento de capital, trabajo y bienes mediante transferencias, migraciones y comercio, se afirma como la condición primaria para la obtención de un mejor nivel de vida. El concepto alternativo de frontera, que Hansen tiende a evadir, sugiere una entidad territorial, con vida política propia.

Hansen es cuidadoso de no utilizar una cxpresión tan fuerte como "artificial" al referirse a la línea limítrofe entre México y los Estados Unidos, aunque de hecho está implícita. El habla de "rclaciones simbióticas" entre Estados Unidos y México y de una "creciente interdependencia" como resultado de las relaciones comerciales y financieras. ${ }^{8}$ Incluso menciona con aprobación la sugerencia de Joel Garreau de una nueva y políticamente autónoma región llamada Mex-América que abarca muchos de los estados del suroeste de Fstados Unidos y el norte de México, la que para fines de siglo, será la "dominante y más poblada región de Norteamérica. . "9 Desde esta perspectiva, los viejos límites políticos habrían perdido su kazón de ser. Rediseñando el paisaje nacional se pretende que los factores económicos hablen más poderosamente que los acuerdos políticos.

\footnotetext{
$\overline{8}$ Idem.

${ }^{9}$ Garreau, Joel, 1981.
} 
Autonomía regional. El argumento de Hansen de autonomía regional comprende varias partes:

a) Las complementariedades económicas y sociales entre ciudades hermanas en la frontera México-Estados Unidos, así como los frecuentes efectos "desbordan

a) Las complementariedades económicas y sociales entre ciudades hermanas en la frontera México-Estados Unidos, así como los frecuentes efectos "desbordantes", han conducido a esfuerzos para la solución de problemas en asuntos tales como control de incendios, aplicaciones legales, educación y salud los cuales, según nos informa Hansen, cumplen con la descripción previa de ser "sumamente informales y por tanto fragmentarios y asistemáticos". ${ }^{10}$ Hansen considera csto como una situación subóptima. En su lugar, èl imagina un proceso amplio que va más allá del intercambio de información y de la consulta recíproca hacia un modelo de "cooperación sistemática" y de "armonización" de la planeación fronteriza. ${ }^{11}$ "Para la mayoría", escribe, "a las autoridades locales y élites creen en lo que es bueno para una ciudad es bueno para la otra. . ."12 $\mathrm{Si}$ al menos actuaran más eficientemente.

b) La región fronteriza está lejos de los centros nacionales de poder. Por tanto, estamos obligados a creer que los problemas de la frontera son inadecuadamente comprendidos tanto en Washington, D. C. como en la ciudad de México, D.F. ${ }^{3}$ No sólo son burocracias nacionales distantes del escenario de los hechos, sino demasiado preocupadas por el "protocolo diplomático y el orgullo por la soberania nacional". ${ }^{14}$ Lo que se propone, entonces es un arreglo internacional que "minimice las dificultades creadas por burocracias del gobierno central" y que "facilite una cooperación transfronteriza relativamente libre para un amplio rango de problemas locales que trascienden las fronteras". 15

c) Tal esfuerzo sería facilitado por metas compartidas y una cultura común en ambos lados de la frontera que ahora parece cada vez más inhibir las "propensiones naturales" y por ende irracional. De acuerdo con Hansen (quién, a su vez, cita un memorándum europeo de Raymundo Strasoldo), las experiencias europeas indican que "la fuerza básica que dirige la interacción tranfronteriza y la cooperación es el desarrollo económico" aún cuando

\footnotetext{
$\overline{10}$ Hansen op. cit.

11 Idem.

${ }^{12}$ Idem

13 Idem.

$14 \mathrm{Idem}$.

15 Idem.
} 
"la presencia de una base étnica común es un factor favorable".16 " $\mathrm{Ambas}$ condiciones", concluye Hansen, "existen a lo largo de la frontera MéxicoEstados Unidos". Del lado norteamericano, la población mexico-norteamericana se sitúa alrededor del 92\%/o) mientras que en lado mexicano, el dólar americano se ha convertido en un medio común de cambio. En cada par de ciudades en la frontera, las élites negociantes locales sueñan con un crecimiento deshinhibido que se relaciona con fronteras abiertas.

Cooperación económica internacional. Basado en su estudio de las experiencias de Europa Occidental, Hansen nos habla de los beneficios mutuos que resultan del libre flujo de capital y trabajo a través de las fronteras internacionales; de la cooperación económica entrc países adyacentes en los ámbitos de política pública y plancación; y de la transustanciación de los que fucron problemas políticos sin una solución clara, en problemas técnicos para los que las soluciones estén a la mano. La cooperación internacional entre países vecinos es por tanto una respuesta razonable.

"Dentro del Consejo de Europa, las autoridades locales y regionales relevantes han tenido finalmente un éxito considerable en convencer a los gobiernos centrales de que la cooperación implica beneficios mutuos para los participantes y que no amenaza la soberanía nacional". 17

$\mathrm{La}$ interdependencia económica $-\mathrm{y}$, en el caso de México y Estados Unidos, la interdependencia es verdaderamente (masiva)- implica principalmente mercados mexicanos, recursos agrícolas mexicanos, trabajo mexicano, y deudas internacionales. ${ }^{18}$ Aunque Hansen no menciona los detalles relevantes, claramente favorece un tipo de acuerdo de mercado común que tendría a la economía de EE. UU., como su pieza central. ${ }^{19}$ Parece particularmente influenciado por el trabajo del Consejo de Europa 20 que facilita la cooperación transfronteriza para un amplio rango de asuntos tal como se concibe, esta forma de cooperación estaria basada localmente pero coordinada mediante un marco binacional que reflejará los intereses "armonizados" en cuestiones cconómicas.

Con esta visión de una comunidad económica norteamericana basada en el modelo europeo, el círculo del argumento se cicrra. El artificio de límites políticos es considerado obsoleto por las fucrzas económicas que, liberadas ahora de las restricciones políticas, son libres para reestructurar el espacio económico a través del continente.

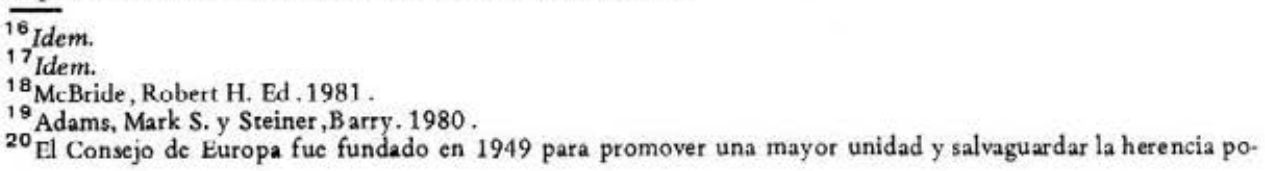




\section{LAS FALACIAS EN EL ARGUMENTO}

Los argumentos de Hansen frecuentemente tienen eco en observadores norteamericanos. Realmente, en su elaboración, ćl se apropia libremente del trabajo de éstos. ${ }^{21}$ Es una perspectiva de mercado libre, y como tal, es la perspectiva del más poderoso.

En asuntos internacionales, los diseñadores de la política norteamericana invariablemente defienden el mercado libre, lo que a su vez, asegura el libre flujo de capital americano (y la correspondientes captación de capital externo), la penetración de mercados externos por mercancías norteamericanas, y la circulación libre de ideas norteamericanas e ideología por medios controlados finalmente por intereses corporativos centrados en los Estados Unidos. Los beneficios de mercados libres por tanto siempre son para el más fuerte. Y quién es el más fuerte es, en este caso, de todos conocido. Para 1970, el comercio de México con los FE. UU. representó dos terceras partes de la totalidad de su comercio exterior; para los Estados Unidos, en relación a su total, este comercio escasamente alcanzó un $3 \% \mathrm{o}^{22}$

Debemos ahora puntualizar en algunas de las deficiencias principales en el argumento de Hansen.

1. Las fronteras son resultados históricos, no artificiales. Las fronteras limitan países que poseen integridad propia, sus propios sistemas políticos y legales, y su propia historia como naciones. La frontera, como resultado entre Estados Unidos y México, muestra una herencia de amargas luchas sobre derechos territoriales. Perdido en la memoria de los EE. UU., alguna vez México se extendía sobre la mayor parte del Oeste norteamericano hasta el paralelo 42. En una sucesión de maniobras políticas y guerras agresivas, los Estados Unidos llevaron la frontera atrás hasta su actual localización en cl mapa. No hay razón para suponer, de cualquïer modo, que ésta se haya fijado permanentemente y que no pudiera, llevarse de nuevo, más al Sur, cncubicrta por los argumentos ostensiblemente no-políticos y "objetivos" de libre comercio y solidaridad fronteriza. La invención de Joel Garreau de Mex-América (1980) es una ingeniosa fantasía según la cual todavía otra parte de México sería incorporado a una economía norteamericana en expansión bajo la hegemonía del capital internacional.

Lejos de ser resultado de un mero capricho político, la frontera separa, de hecho, dos sistemas estatales y legales sumamente diferentes que están basados en diferentes tradiciones. La planeación es parte de esos sistemas. Y

\footnotetext{
lítica y cultural en Europa.

21 Ver los trabajos de Sloan y West, de 1976 y 1977 ,asi como los de Stoddard, 1979 y Clement, N. 1982.

22 Adams, op. cit. p. 375.
} 
cuando Hansen apasionadamente defiende un mayor grado de autonomía regional y local, se está refiriendo principalmente al Estado mexicano en su tradición de instituciones centralizadas. ¿No podría ser que la descentralización del poder en México - lo que Hansen ve como un ingrediente esencial de una cfectiva política de planeación fronteriza- tuviera éxito simplemente en abrir la senda para un mayor avance de corporaciones norteamericanas hacia el sur bajo el pretexto de libre comercio?

Cualquier cosa que pueda uno pensar al respecto, es claro que la frontera es algo más que una simple línea en el mapa. ${ }^{23}$ Es un arma en la lucha de intereses nacionales y puede utilizarse tanto ofensiva como defensivamente, como se muestra en el cuadro I.

\section{CUADRO I. USOS POLITICOS DE LA FRONTERA MEXICO-ESTADOS UNIDOS.}

\begin{tabular}{|c|c|c|c|}
\hline Usos of & isivos & & sos defensivos \\
\hline E.U.A & México & E.U.A. & México \\
\hline $\begin{array}{l}\text { Doctrina del } \\
\text { libre comer- } \\
\text { cio. }\end{array}$ & $\begin{array}{l}\text { Apertura de la frontera con } \\
\text { E.U. a migrantes mexicanos } \\
\text { que deseen laborar en E.U. }\end{array}$ & $\begin{array}{l}\text { Control de la mi- } \\
\text { gración laboral } \\
\text { desde México y } \\
\text { el resto de Amé- } \\
\text { rica Latina. }\end{array}$ & $\begin{array}{l}\text { Uso de tari- } \\
\text { fas para pro- } \\
\text { teger la in- } \\
\text { dustria na- } \\
\text { cional de la } \\
\text { competencia } \\
\text { de E.U. }\end{array}$ \\
\hline $\begin{array}{l}\text { Visión agre- } \\
\text { siva para la } \\
\text { apertura de } \\
\text { la economía } \\
\text { mexicana al } \\
\text { capital nor- } \\
\text { teamericano } \\
\text { sin restric- } \\
\text { ciones. }\end{array}$ & $\begin{array}{l}\text { Establecimiento de EPZ (es- } \\
\text { pecialmente sobre la fronte- } \\
\text { ra con tributación y repa- } \\
\text { triación de beneficios } \\
\text { y otras ventajas). }\end{array}$ & & $\begin{array}{l}\text { Estímulo a } \\
\text { la producción } \\
\text { nacional. } \\
\text { tanto para el } \\
\text { mercado in- } \\
\text { terno como } \\
\text { de exporta- } \\
\text { ción median- } \\
\text { te devalua- } \\
\text { ciones del } \\
\text { peso. }\end{array}$ \\
\hline
\end{tabular}

2. La cooperación transfronteriza de Europa Occidental es un modelo inapropiado para las relaciones México-Estados Unidos. Los ejemplos de Europa Occidental sobre cooperación transfronteriza, tan apreciados por Hansen, son una analogía inapropiada de la situación en la frontera México${ }^{23}$ Brown, Peter G. y Shue, Henry, 1981. 
Estados Unidos. La mayor parte de los ejemplos mencionados por Hansen se refieren al Valle del Rhin, que corre del centro financiero de ZurichBasilea al puerto de Rotterdam en Holanda. Esto es el corazón de la Europa industrial que abarca ciudades que desempeñan un papel crucial como centros de control financiero, investigación, dirección y producción industrial altamente calificada. Es una región económica contínua en la que destacan importantes centros urbanos dentro de 50 millas respecto a su eje principal: además de los mencionados, Estrasburgo, Mannheim, Frankfurt, Bonn, Colonia, Dusseldorf, Essen, Dortmund y Randstadt, en Holanda situada entre Rotterdam, Amsterdam y Utrech. Los países vecinos de Suiza, Alemania Occidental, Francia y Holanda están entre los más ricos en el mundo, y aunque de extensión desigual, han aprendido a integrar sus economías regionales como socios en la asamblea europea de naciones.

En contraste, la mayoría de las ciudades de la frontera México-Estados Unidos son pequeñas y relativamente poco importantes nacionalmente y seguramente menos internacionalmente. En muchos casos, son una creación original de la frontera misma, y tienen escasa razón de existir excepto como puntos de tránsito de un país a otro. Vinculando los principales mercados y centros de población de ambos países, su orientación dominante es NorteSur; el comercio horizontal entre ellas es mínimo y sus economías locales tienen muy poco que ofrecer entre sí. Desde un punto de vista económico y cn un marcado contraste con el Valle del Rhin, la frontera México-Estados Unidos no es una "región" en sentido económico. Scpara dos economías nacionales sumamente diferentes y de distinto desarrollo. Veamos algunas de las dimensiones contrastantes. ${ }^{24}$

- Los estados Unidos tienen tres veces la población y cerca de cinco veces el área de México. Su Producto Interno Bruto (PIB) es doce veces mayor que el de México.

- El ingreso per cápita en Estados Unidos es cerca de seis veces el de México Un indicador más sugerente es el nivel de consumo de energía per cápita que, en Estados Unidos, es siete veces más alto que en este país vecino.

- La tasa de crecimiento poblacional en los Estados Unidos es sólo un tercio de la de México, pero la mortalidad infantil en México es cuatro veces más alta, en tanto que la esperanza de vida al nacer es nueve años menor que en los Estados Unidos.

- El ingreso está más inequitativamente distribuído en México, donde el 20\% más bajo de la población recibe sólo el 2.9\% del ingreso total (contra $4.5 \%$ de todos los ingresos (contra 43\% o en los Estados Unidos).

$\widetilde{{ }^{24} \text { Banco Mundial, } 1983}$ 
- Los países tienen una contribución similar del sector manufacturero al PIB (22 contra 230/o). Pero en México, un tercio de toda la producción industrial es controlada por corporaciones transnacionales $y$, en algunos sectores, como los bienes de consumo durable, el porcentaje es mucho más alto (los datos de corporaciones a 1970 . Actualmente, la proporción de capital controlado por extranjeros en México debe ser bastante más alta).

- En México, el 36\% de la fuerza de trabajo aún trabaja en actividades agrícolas; en los Estados Unidos la proporción correspondiente es de sólo $2 \%$.

- Los contrastes en los niveles educativos de la población son severos: población adulta alfabetizada, 83 contra $99 \%$ o; cscolarización secundaria, 37 contra 97\% o cducación supcrior, 15 contra $55 \%$ o.

¿Qué conclusiones podemos derivar de estos datos conocidos? Los problemas y prioridades para la acción pública, son claramente muy diferentes para estos dos países. Mientras que la economía de los Estados Unidos pertenece al núcleo del sistema internacional, México es parte de la llamada semiperiferia, que está funcionalmente subordinada a ese núcleo. En la solución de estos problemas nacionales comunes a los países semiperiféricos, México está obligado a utilizar su frontera norte defensivamente. Sólo de esta forma es posible obtener la flexibilidad requerida para realizar los ajustes a la política interna que posibiliten un patrón nacional de desarrollo.

3. Interdependencia económica no implica armonía de intereses: la Comunidad Norteamericana es una ilusión. Hansen supone que la experiencia europea de cooperación "implica beneficios mutuos para los participantes y que no amenaza la soberania nacional". ${ }^{25}$ El Consejo de Europa afirma que las naciones miembros de la Convención Limitrofe Europea están "dispuestos a promover esa cooperación tanto como sea posible y contribuir de esta manera al progreso social y económico de las regiones fronterizas y al espíritu comunitario que unc a los pucblos de Europa". ${ }^{26}$ Pero la historia de las relaciones entre México y Estados Unidos ofrece muy poco de tales expectativas. Tan reciente como la crisis petrolera de los setentas, las rupturas parecen más importantes de lo que fueron intereses armoniosos. Derivadas de posiciones políticas tanto liberales como conservadoras en los Estados Unidos, han surgido propuestas cuidadosamente diseñadas para un mercado común norteamericano, que conducirían simultáneamente a una mayor integración económica de Canadá, los Estados Unidos y México como al acceso directo de Estados Unidos al petróleo de sus vecinos. Ni Canadá ni

\footnotetext{
${ }_{26}^{25}$ Hansen, op. cit.
${ }^{26}$ Idem.
} 
México han aprobado esta idea. La abrumadora oposición de los mexicanos es resumida por Carlos Rico F.: ${ }^{27}$

- La idea de un mercado común es el primer paso para hacer de México más una parte de los Estados Unidos que un socio. En este sentido, la propuesta ha sido llamada una "provocación sofisticada" de México.

- Segundo, a pesar de las benévolas declaraciones que enfatizan las preocupaciones generales de las dos (¿tres?) partes involucradas en tal "comunidad", las propuestas más articuladas huelen a petróleo. En lugar de ver esas propuestas como un paquete general de cooperación, los observadores mexicanos las han visto como vehículos para los Estados Unidos para tener acceso a los recursos energéticos mexicanos.

- Tercero, aquellas versiones de la propuesta que enfatizan en el intercambio de biencs y servicios son en realidad mecanismos para "reciclar" "petropesos" mexicanos. México se vería estimulado a importar indiscriminadamentc, incluyendo productos que en muchos casos no son necesarios.

- Cuarto, los supuestos de libre movilidad de factores de la producción (p. e. trabajo), un requerimiento básico para los supuestos económicos del modelo, no son una posibilidad viable frente a la realidad política de corto y mediano plazo de los EE. UU.

- Quinto, aplicar la teoría de las ventajas comparativas bajo tales restricciones, podría conducir a México a especializarse en aquellos productos de los que han intentado liberarse en los últimos veinticinco años.

- Finalmente, mientras que los exponentes norteamericanos de la retórica de la interdependencia han estado ampliamente en favor de la idca de un mercado común, los exponentes mexicanos de la misma retórica la han rechazado aunque por diversas razones.

No hay razón para creer que la propuesta de Hansen pudiera ser más benevolente. Al contrario, con su requisito de un cambio en la sede de la toma de decisiones del nivel nacional al regional, el control político mexicano sobre las fuerzas económicas globales quedaría irrevocablemente disipado. ¿Qué intereses se verían favorecidos con tal movimiento? En lugar de coincidir con Hansen, nos situamos al lado de los críticos de la reciprocidad, los intereses compartidos y el difícil objetivo de "equilibrio", sosteniendo que la nación más poderosa del mundo no puede hacer pareja con una nación en desarrollo, aún bajo la rúbrica de la planeación para una "región"

$\overline{27}$ En McBride, op. cit. 
fronteriza, y pretender que el resultado pueda ser aquel visualizado por las naciones industrializadas de la Europa Occidental.

4. Los esfuerzos informales de planeación transfronteriza no son inherentemente ineficientes. Hansen se refiere a las prácticas actuales de la planeación en la frontera como "fragmentarias y asistemáticas". Puede ser, aunque el hecho es apenas afirmado. ¿Existe alguna razón por la que debiéramos suponer que la forma idealmente deseada de planeación es ordenada y comprensiva? La mayoría de los planificadores y teóricos de la planeación hace tiempo que abandonaron el modelo de reacionalidad sinóptica. No era aplicable, dijeron. Para reemplazarlo proponen una forma de planeación de ajuste mutuo, mientras que otros propugnan por un modelo de aprendizaje social. ${ }^{28}$ Por tanto, establecer que las soluciones en la planificación deben ser vistas como problemas originados por (sí solos) no es reprensible ni equivocado. Esto no es un elogio de lo ad boc, tampoco un reclamo de que sólo la informalidad es adecuada. Sin embargo, los procedimientos informales son a veces la manera pragmática de solucionar problemas comunes y no deben ser en conjunto desechados por falta de reacionalidad. Las fallas en la comprensión sistemática son demasiado conocidas para brindar un juicio fácil sobre el asunto.

5. Los intereses de las élites no son los únicos intereses que importan. Hansen repetidamente habla de planificar por y para las élites. Tiene en mente a los comerciantes locales, industriales y funcionarios públicos. En ambos lados de la frontera, dice, están a favor del desarrollo económico por encima de otros objetivos. Y los cita diciendo: "lo que es bueno para una ciudad es bueno para la otra". La pretensión de las élites de hablar a nombre de todos, de nuevo descansa en el conveniente supuesto neoclásico de que no hay diferencias fundamentales de intereses entre clases y grupos, y que las élites simplemente articulan el consenso social existente.

Si este supuesto no fuera tan frecuente y estridentemente afirmado y reafirmado, uno difícilmente se molestaría en refutarlo. Como todos sabemos, existe desacuerdo y la sociedad no es homogénea, la gente valora cosas diferentes, las élites sólo hablan por ellos. Si algunos grupos específicos, géneros o intereses de clase coinciden o nó es una cuestión a determinar empíricamente. La pregunta no puede ser tratada como si fuera universalmente reconocida, como principio a priori.

6. Una mayor autonomía regional no es más deseable que los intereses regionales. Hansen empequeñece el interés nacional. Leyéndolo, uno puede conjeturar que no hay nada más consecuente que el protocolo diplo-

\footnotetext{
${ }^{28}$ Para una historia critica del pensamiento planificador, ver: Friedman, 1984.
} 
mático (una simple formalidad) y una cuestión de "orgullo nacional". Las élites locales, dice Hansen, "se sienten vulncrables a los caprichos de quienes determinan las políticas en sus respectivas capitales nacionales". ${ }^{29} \mathrm{Y}$ aún así, es fácil ver cómo hemos tratado de demostrar que la frontera puede, de hecho, ser utilizada de manera bastante diferente por diferentes Estados, y que, en el caso de México y Estados Unidos, los usos ofensivos y defensivos no tienen sólo claras diferencias de intención, sino que también se preocupan de preguntas como la movilidad de capital y trabajo, que son de interés vital para los dos países, aunque su significado e importancia sean diferentes para cada uno. En México específicamente, donde existe una tradición centralista de gobierno, no debe suponerse automáticamente que la descentralización del poder del Estado a las autoridades locales sea efectiva, particularmente cuando estas últimas se enfrentan a la presión del capital transnacional y al mucho más sustancial control fiscal de sus contrapartes norteamericanos.

\section{RESUMEN Y CONCLUSION}

En nuestra refutación de la postura de Hansen sobre una formalización de la planeación transfronteriza mediante un acuerdo internacional basado regionalmente entre México y los Estados Unidos, se adelantaron los siguientes argumentos:

1. Las fronteras son resultados históricos, no construcciones artificiales.

2. La cooperación transfronteriza de Europa Occidental es un modelo inapropiado para las relaciones México-Estados Unidos.

3. Interdependencia económica no implica armonía de intereses; la Comunidad Norteamericana es una ilusión.

4. Los esfuerzos informales de planeación transfronteriza no son inherentes ineficientes.

5. Los intereses de las élites no son los únicos intereses que importan.

6. Una mayor autonomía regional no es más deseable que los intereses nacionales.

Aquí debemos preguntar si las alternativas al modelo regional propuesto por Hansen scrían preferibles. Nuestra respuesta es que ninguna solución única es suficiente: la complejidad de la frontera México-Estados Unidos re-

\footnotetext{
$\overline{{ }^{29} \mathrm{El}}$ subrayado es del autor.
} 
quiere de una sensibilidad sin paralelo histórico reciente. Más aún, creemos que segmentos y estratos más amplios de la población, más que meras élites, deben incorporarse al proceso de planeación existente. El asunto de la coope ración bi-nacional requiere algo más que la precipitación hacia una tendenciera forma de autonomía regional y planeación transfronteriza como la que propone Hansen,

\section{BIB LUOGRAFIA}

ADAMS, Mark. S. and Barry Steiner (1980). "Energy and the North American Community: Canada, México, and the United States," The Hastings international and compa ative law review, 3, 3 (Spring), 369-434.

BROWN, Peter G. and Henry Shue, eds. (1981). Boundaries: National autonomy and its limits. Totowa, N.J.: Rowman and Allanheld.

CLEMENT, Norris (1982). United States border relations: History, issues, and institution. Unpublished paper presented at the March 17-19 Bi-national American Assembly on the U.S. México Bordtrlands, El Paso, Texas. San Diego: San Diego State University.

FRIEDMANN, John. (1984). Planning in the public domain A critical history, Los Angeles: UCLA, Graduate School of Architecture and Urban Planning.

HANSEN, Niles (1983). "European Transboundary Cooperation and Its Relevance to the United States-Mexico Borders," Journal of the American Institute of Planners, 49, 3 (Summer), 336-343.

McBRIDE, Robert H. ed. (1981) México and the United States: Energy, trade investment, immigration, fourrism. Para la Asamblea Americana, ver Englewood Cliffs, N.J.: Prentice Hall. Carlos Rico F., "The future of Mexican-U.S. relations and the limits of the thetoric of "interdependence" en Carlos Vasquez y Manuel Garcia y Griego, Mexican.U.S. Relations: Conflict and convergence. Los Angeles. Calif.: Chicano Research Center Publications, UCLA, 1983

SCHTEINGART, Martha y Gustavo García (1984). México City: Industrial dynamics and the formation of space in a semi-peripheral metropolis". Manuscrito sin publicar. 